\title{
Simposium sobre Esterilidad Matrimonial
}

\begin{abstract}
Trabajo presentado por el Departamento de Ginecología, Sección de Esterilidad, del Hospital San José, al Primer Congreso Colombiano de "OBSTETRICIA Y GINECOLOGIA".

Jefe del Departamento: Prof. DANIEL BRIGARD H., Jefe del servicio de Esterilidad DR. ARTURo APARICIO, Profesor titular de Clinica Ginecológica. Miembro de The International Fertility Asociation.
\end{abstract}

Cuando a mediados del año próximo pasado, quisimos iniciar el "Consultorio de Esterilidad", acudimos al Hospital de San José ,casa siempre abierta a todas las inquietudes científicas y colaborador eficaz en todo lo que atañe al mejoramiento de sus servicios o que redunde en provecho de la investigación o del adelanto de la medicina nacional. Con amplias facultades para organizarlo dependiente del Departamento de Ginecología y anexo a la consulta externa del Hospital San José, creamos este servicio, que si pobre en dotación material, es rico en entusiasmo y en elemento humano, deseoso de prestar con su colaboracion, un valioso aporte a la especialidad y a la clase social más necesitada. Y al hablar de la clase social más necesitada, no lo hacemos por puro formulismo, sino que en realidad de verdad, la consulta cada vez mayor de matrimonios modestos que acuden a los especialistas con el objeto de solucionar su problema de falta de descendencia, nos obliga a pensar, no ya con un criterio meramente investigativo, sino como una necesidad social que deben solucionar nuestras instituciones hospitaliarias.

En este orden de ideas y para no ponerles sino un solo ejemplo de nuestra experiencia ,el Consultorio de Esterilidad del Hospital San José, ha recibido y estudiado en el curso de seis meses de existencia 100 pacientes. Esta cifra, bien diciente por cierto de nuestro acerto, nos indujo, a presentar a la consideración del Primer Congreso de OBSTETRICIA Y GINECOLOGIA, la organización dada al consultorio de ESTERILIDAD del Hospital San José, en la seguridad que de su estudio, críticas o comentarios, podamos sacar enseñanzas útiles para todos. Sobra decir, que las puertas del servicio están abiertas para todos y que muestro mayor anhelo sería el despertar en este Congreso las inquietudes sobre estos problemas y el de trabajar en colaboración con los colegas, ya sean locales o de provincia, que quieran honrarnos con su presencia. 


\section{Organización de un Consultorio de Esterilidad}

Planteamiento del problema: El problema de la falta de descendencia hu mana, constituye una consulta que a diario se ofrece al especialista y ya que no es dable hablar hoy dia, de esterilidad temenina o de esterilidad del varón, el ginecólogo moderno, dedicado anteriormente al estudio exclusivo de la mujer, tendrá que adentrarse en el campo masculino, para poder estudiar y resolver e] problema.

Resultaria absurdo, según nuestro criterio, fijar, mediante una estadística rigurosa el tanto por ciento de veces que reside en el varón o en la.mujer la causa de la esterilidad. A excepción de aquellos casos en los que se encuentra una causa obsoluta de esterilidad (azoospermia en el varon, oclusión tubárica en la mujer), en la mavor parte de los matrimonios que llegan a nuestros consultorios planteándonos el problema, descubrimos la existencia, no de uno, sino de varios factores depresores de la fertilidad, causas en realidad relativas y que afectan tanto a la mujer como al hombre. Esa es la razón, por la cual, al hablar del problema, debemos hacerlo, no con criterio unilateral, sino de conjunto bajo el apelativo de "Esterilidad conyugal o matrimonial".

Poseemos en verdad, datos ciertos que nos permiten establecer quc las posibilidades generativas de la pareja humana están determinadas por la relación existente entre la potencia generativa (fertilidad) de ambos cónyugues, de una parte, y de otra, por la suma de factores depresores de la fertilidad, causas relativas, que afectan tanto al varón como a la hembra. Esa la razón por la cual observamos con sorpresa en nuestros consultorios, la disparidad de resultados en relación con la fecundación que muchas-veces nos sorprende. Cuántas veces hemos observado que una mujer con una lesion genital insignificante, no logra ser fecundada por un marido potencialmente fertil, en tanto que logramos un embarazo en un matrimonio en el cual el marido tiene, evidentemente, un poder fecundante relativamente bajo.

A la luz de está concepción, nos explicamos también perfectamente y sin necesidad de recurrir a interpretaciones un tanto hipoteticas, como la de la incompatibilidad biologica (estado de inmunidad o de alergia de la mujer por el semén del marido) el hecho por todos los autores referido, de que éstos mismos cónyugues consigan descendencia en un nuevo matrimonio. Quiere esto decir, que el nuevo cónyugue viene a compensar con su gran fertilidad, los defectos fertilizantes del que, en su matrimonio anterior. no tuvo esta compensación y se quedó sin hijos.

Es esta la razón por la cual, ya no es posible emprender una investigación diagnóstica causal en los casos de esterilidad. prescindiendo del estudio de uno de los cónyugues. Al hacer la investigación aislada, podemos encontrar muchas veces las causas que justifiquen la falta de descendencia: pero sería poco cientítífico y muchas veces pozo honrado, el prescribir tratamientos sin el conocimiento previo del poder generador del otro convugue. 
Planteado el problema en esta forma, vemos que para llegar al diagnóstico preciso de una esterilidad conyugal, no sólo se requiere el conocimiento anatomofisiológico, por así decirlo de la pareja, sino que entran en juego una serie de factores que es preciso estudiar e investigar cuidadosamente. Para llegar a ese objetivo se requiere estar en posesión de ténicas especiales que permitan estudiar los diversos factores que en un momento determinado puedan presentarse y de ahí la necesidad absoluta de buscar colaboración con el urólogo, el radiólogo, el endocrinólogo, el laboratorista o con el histopatólogo, para la cabal aclaración del problema. Tal serie de exámenes, gravosos la más de las veces para gran parte de la clientela, obligan forzosamente a la pareja a descontinuar las investigaciones y a perder, en muchas ocasiones por falta de estudio, la anhelada concepción. Una razón de más, para que el problema se convierta en eminentemente social y de ahí nuestro entusiasmo, para que, al presentar a la consideración de ustedes nuestro modesto plan de trabajo, nos veamos muy pronto acompañados en nuestra labor por el mayor número de instituciones particulares u hospitalarias, en todo el territorio del país.

Empero, para realizar este objetivo, no sólo se requiere el conocimiento esmerado en determinadas téenicas y en la organización de equipos, material y humanamente capacitados, sino en la constante y paciente labor de educar a nuestros hombres. La especial idiosincrasia de nuestra raza, y la manera primitiva como se entienden todavía estos problemas, no sólo por parte de nuestro público ino también por parte de muchos profesionales, hacen que dificulte o se demore considerablemente nuestro empeño. Por una u otra causas, el marido, que tiene el errado concepto de creer que la potencialidad es sinónimo de fertilidad, achaca casi siempre el insuceso a la mujer, rehusando en un gran número de casos su examen físico completo. Nuestras historias de Hospital, adolecen en gran parte de esta falla, que hemos venido subsanando lentamente a base de educación y de facilitar los exámenes en horas cómodas para las clases trabajadoras. En nuestra consulta particular ,rehusamos en cambio, sistemáticamente, estudiar un caso de esterilidad cuando el marido rehusa someterse a los exámenes de rigor. Muchas veces aceptamos la conseja de que el marido era fértil so pretexto de haber concebido hijos en otra mujer y al encontrar normal por todos conceptos a la esposa e insistir en el estudio del esposo, nos encontramos sorprendidos ante el hecho de que la infertilidad residíd en el marido, que presumía de una paternidad extraconyugal.

Este falso concepto, que ha hecho a los hombres creer en su permanente fecundidad, ha obligado a las mujeres acicateadas por su instinto de la maternidad o apremiadas por circunstancias especiales de su vida conyugal, a plantear al médico el problema de su falta de descendencia. Casi siempre llegan a los consultorios acompañadas, no del esposo, sino de la madre o de una amiga, simulando cualquier molestia, para indagar la causa de su esterilidad. El mé dico, por su parte, debe tener la suficiente perspicacia y comprensión para encarar el problema en todos sus aspectos y una vez hechas las exploraciones de rigor, no comprenderá, en conciencia, tratamiento alguno encaminado a curar 
la esterilidad, sin haberse percatado previamente de la buena fertilidad del esposo.

Si se descubre alguna lesión genital o algún trastorno funcional que la mujer desea tratarse, estamos, naturalmente, autorizados para hacerlo, advirtiendo, que sólo pretendemos curar la ginecopatía y no la esterilidad. Lo que no debc hacerse en manera alguna, es probar tratamientos de esterilidad en mujeres sin descendencia y en las que, el reconocimiento ginecológico no descubre aparentemente ninguna causa que explique la esterilidad matrimonial. Doloroso es el caso observado por nosotros, no hace mucho tiempo, de una pobre mujer que después de haber sido tratada por varios colegas durarte tres años, encontramos en el marido una criptorquidia con ausencia absoluta de espermatozordes.

Sentados estos conceptos de carácter general, queremos aclarar los conceptos en relación con las palabras mentadas tantas veces en esta clase de trabajos: Esterilidad e Infertilidad. En realidad y de acuerdo con la mayoría de los autores, estos términos, no son sinćnimos. Por Esterilidad, debemos entender en la mujer. la incapacidad o imposibilidad de concebir de un modo más o menos permanente; mientras que por Infertilidad debemos entender "la imposibi. dad tempora! para concebir o la imposibilidad de llevar a cabo estas concepciones". En el primer caso se trata, por consiguiente, de la imposibilidad material de conjunción de ambos gametos, en tanto que en el segundo, es decir, en la infertilidad, el ovulo fecundado, es decir el huevo, al encontrar condiciones desfavorables para su implantación o desarrollo perece precozmente, desintegrándose $\mathbf{y}$ siendo absorbido rápidamente o bien expulsado al exterior antes de tiempo (áborto espontáneo o "diskiesis") (Ahumada). Como se ve, en ambos casos fracasa la tentativa de procreación, siendo por otra parte muy difícil determinar si se trata en realidad de un caso absoluto de esterilidad o de regresión precoz de un órulo. En la práctica estos dos términos se confunden, no pudiendo ha. blar entonces sino de Esterilidad absoluta, temporal o relativa.

La esterilidad absoluta es aquella que se observa en los grandes síndromes genitales tales como azoopermia en el hombre, o agenesia orarica o tubárica en la mujer. La esterilidad temporal es en cierto modo fisiológica y se comprueba en la mujer en los siguientes estados:

10-Antes del completo desarrollo femenino, es decir, entre la nubilidad y la pubertad (Botella, Ashley-Montagu).

20-Periódicamente, todos los ciclos, después del tercer día de la ovulación y hasta cuarenta y ocho horas antes de la orlución siguiente (Knaus).

$3^{\circ}$ - A partir de una cierta edad, que puede en término medio fijarse desde los cuarenta y dos a los cuarenta y cuatro años, aun cuando sigan apareciendo reglas de características más o menos normales. (Esterilidad premenopáusica).

4-Durante la lactación (al menos en muchos casos) y

$5^{\circ}$-Según DIDDLE y colaboradores, en mujeres perfectamente normales, sin que se sepa por qué, en determinadas épocas de la vida o estaciones del año. 
La esterilidad relativa, se confunde a nuestro entender con la infertilidad y son aquellos casos en que intervienen factores depresivos de diversa índole y naturaleza, pero que están a nuestro alcance para estudiar y muchas veces, para tratar satisfactoriamente.

La Infertilidad podriamos compararla a la manera de un terreno que para producir convenientemente sus frutos, necesitara abonarlo y prepararlo. En este sentido, el término de Infertilidad es mucho más comprensivo y amplio que el de Esterilidad, pues abarca, no sólo este último término, sino también, la falta de descendencia por aborto de repetición, por parto con feto muerto o la falta temporal de descendencia como ocurre en la pubertad y en ciertos momentos de la vida femenina.

A pesar de esta discriminación el término Esterilidad, aceptado por la fuerza de la costumbre, es, hasta la fecha el imperante, entendiendo, que al hacer su estudio, no vamos a luchar contra lo imposible (esterilidad en el sentido nato de la palabra), sino contra aquellas causas de infertilidad, que por una u otra causa estén perturbando la fecundidad.

Como quiera que muchas mujeres son estériles temporalmente de un modo fisiológico, no podemos definirla como estéril, sino pasado cierto tiempo de matrimonio y sin descendencia. Por lo general, nuestras mujeres apremiadas por el afán de concebir un hijo o aconsejadas por sus parientes o amigas, acuden al consultorio del especialista en plazo relativamente corto después de su matrimonio. Nuestro consejo en tales casos, y previo el examen ginecológico cuidadoso para descartar las grandes causas anatomicas, se limita a aconsejar a la paciente un compás de espera prudencial, que de acuerdo con los autores varía entre uno y dos años, advirtiéndole que antes de ese plazo puede ser perjudicial el adelantar tratamientos que por lo intempestivos, pueden serle francamente contraproducentes. Debemos, sí, darle algunas normas o consejos prudentes y medidos en relación con su nuevo estado, ganándonos de este modo su confianza y preparándola en esta forma, para los estudios ulteriores en caso que fueren necesarios.

Estudio de la pareja estéril: En presencia va, de un matrimonio que acude a] especialista con el objeto de tratar un problema de falta de descendencia, el médico debe emprender su estudio de acuerdo con una orientación o plan determinado que le permita aclarar el problema en un tiempo relativamente corto. No quiere esto decir que podamos resolver el problema de inmediato y que las más de las veces debemos advertir a la pareja, sin asegurar el éxito, que los tratamientos son largos y engorrosos.

El plan de estudio que acostumbramos seguir, tanto en nuestra clientela particular como en la hospitalaria, se reduce a estudiar de una manera detallada, los diversos factores que interviene en el desarrollo del problema, factores que podemos dividir de la manera siguiente: 


\section{a) FACTOR GINECOLOGICO EN GENERAL.}
b) " MARIDO.
c) " CERVICAL.
d) " TUBARICO.
e) " OVARIO-ENDOCRINO.
f) " PSICOSOMATICO Y AMBIENTAL.

Cada uno de estos temas, será objeto de cuidadoso estudio por parte del especialista, quien al analizarlos y valorarlos en su justo valor, podrá dar un consejo acertado y emprender un tratamiento hasta donde sea posible, etioló gico y racional.

Captado el problema en esta forma, el plan de investigación que podemos seguir variará de acuerdo con la época o fecha en que la pareja llegue a consultar, fecha que estará siempre en relación con el dato de la última menstruación. Si la pareja llega en la primera mitad del Intermenstruo, podemos en rigor adelantar, de una vez los siguientes exámenes:

a) Examen espermático.

b) Insulflación quimográfica (antes del día 12 del ciclo).

c) Histerosalpingografia (antes del 14 día. No es necesaria en todos los casos.

d) Entre los días 13 y 16 "Determianción de Gonadotropinas", si es necesario).

e) El día 24 ó 26 en adelante, previa abstención del coito, Microlegrado En el consultorio particular, lo practicamos de rutina siempre el primer día de la menstruación.

f) En el 14 ó 15 dia del ciclo, podemos practicar el Test de penetrabilidad de espermios en el moco cervical (Test de Huhner) o estudio de la cristalización del moco cervical (Test de Roland).

g) Iniciar el estudio de citología vaginal.

h) Enseñar a manejar la gráfica de temperatura basal.

Si la pareja llega al consultorio en la segunda mitad del Intermenstruo, podremos iniciar los siguientes exámenes:

a) Examen espermático.

b) Investigación del Pregnandiol del 22 al 24 día del ciclo.

c) Microlegrado, premenstrual.

d) Persuflación tubárica 4 ó 5 dias después de la reg]a.

f) Del 14 al 15 dia del ciclo, Test de la penetrabilidad del moco cervical.

g) En este lapso de tiempo, y mientras aparece la menstruación, podemos adelantar los exámenes de laboratorio rutinarios, tales como cuadro hemático, curvas de glicemia, Colesterol, metabolismo basal, etc.

Siguiendo estas normas generales, $y$ con equipo de colaboradores eficiente, el estudio de la pareja estéril debe hacerse en el lapso de tiempo comprendido 
entre dos menstruaciones. No se justifica, pues, so pretexto de estudiar una pareja, el hecho por nosotros observado, de clientela que acude a nuestro consultorio después de tres o cuatro meses de investigaciones las más de la veces incompletas y mal orientadas.

Con este orden de ideas, emprendimos la organización del consultorio de Esterilidad del Hospital San José. Autorizados ampliamente por las directivas, llamamos a colaborar un grupo de colegas, ajeno a todo interés pecuniario pero llenos de buena voluntad y deseoso de prestar su valioso aporte de conocimientos en el desarrollo y estudio de tan delicado problema. Sin elementos materiales y pestando cada cual, los elementos necesarios para cada una de las investigaciones, el consultorio es hoy una realidad y ya empieza a dar frutos.

En la actualidad, funciona diariamente de 8 a 10 de la mañana, atendido por una nómina de médicos expertos agrupados en varias secciones, así:

Sección de Ginecología. - A cargo de los doctores: Profesor Agregado Héctor Enrique Bernal, Rodulfo Camero, Alberto Villaneda Soto, Enrique Darnalt Restrepo, Alfonso Rivas Posada y Fernando Tamayo. Nos han acompañado, en calidad de internos, los señores Hugo Quijano y Ricardo Rueda.

Sección de Endocrinología. - A cargo del doctor Antonio Ucrćs.

Sección de Urología. - A cargo de los doctores Alfonso Ramírez y Carlos Perilla.

Laboratorio Clínico: Doctor Hernando Gómez Vesga y colaboradores.

Sección de Histopatología: Doctor Hernando Latorre.

Como hasta la fecha, no nos es posible presentar a la consideración de ustedes un trabajo investigativo especial, resultado del estudio en que estamos empeñacos, hemos creído útil hacer un resumen a la orden del día, de todos los factores fue intervienen en una u otra manera, en el problema, siempre tan novedoso y masionante de la esterilidad.

Cedo, pues, la palabra al "equipo" de médicos colaboradores haciendo púhilico reconocimiento, a nombre del Hospital San José y en el mío propio, por sus laliosos y desinteresados servicios en bien de la Sociedad, de nuestra especialidad $\checkmark$ de la medicina nacional. 